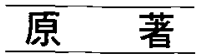

\title{
Dissociation between Eye Movement Range and Velocity Observed in the Course of Fisher's Syndrome
}

\author{
Ken Ito $^{1)}$ - Toshihisa Murofushi ${ }^{1)}$ - Rie Naito ${ }^{2)}$ - Masahiro Mizuno ${ }^{3)}$ \\ 1) Department of Otolaryngology, Faculty of Medicine, University of Tokyo \\ 2) Division of Neuro-otology, Tokyo Metropolitan Neurological Hospital \\ 3) Department of Otolaryngology, Faculty of Medicine, Saitama Medical College
}

Three rare cases are presented that showed a peculiar set of eye movement disorders in the course of Fisher's syndrome, that is, eye movements similar to internuclear ophthalmoplegia, but with restriction of abduction instead of adduction. Characteristics of the abnormal eye movements are summarized as 1) bilateral restriction of the abduction range, 2) bilateral slowing of adducting saccades, and 3) dissociated nystagmus and/or overshooting of the abducting eyes. The possible mechanisms causing these disorders are discussed, and two hypotheses are proposed: 1) possible impairment of inhibition to the medial rectus and 2) independent peripheral control of phasic and tonic eye movements. The latter explanation, primarily assuming peripheral disorders, was considered more suitable.

Key words: eye movement, range, velocity, dissociation, Fisher's syndrome

\section{Introduction}

Internuclear ophthalmoplegia (INO), caused by a lesion in the medial longitudinal fasciculus (MLF) and most common in patients with multiple sclerosis $(M S)^{1)}$, presents a characteristic set of eye movement disorders; impaired adduction of the ipsilateral eye, both in the range and velocity of adduction, and dissociated nystagmus and/or overshooting of the contralateral abducting eye. A set of eye movement disorders resembling INO was reported in myasthenia gravis (MG) ${ }^{23)}$ and Fisher's syndrome ${ }^{45)}$, and is called pseudo-INO because no lesion of the MLF is assumed. These characteristic sets of impaired ocular movements can provide a clue to understanding the mechanisms controlling monocular eye movements, which require for further investigation. In this paper, three rare cases

東京大学医学部耳鼻咽喉科

新専門会員 are presented that showed a peculiar set of eye movement disorders in the course of Fisher's syndrome, i.e., INO-like eye movements in the presence of abduction restriction instead of restricted adduction. The mechanisms causing these disorders are discussed, not excluding possible involvement of supranuclear neurons in Fisher's syndrome ${ }^{677}$.

\section{Case Presentation}

Case 1: Two weeks after a common cold, a 13-year-old girl suddenly noted dysequilibrium and simultaneously became feverish $\left(38^{\circ} \mathrm{C}\right)$. She had received an inoculation of influenza two days before onset. Her past history was not remarkable, nor was her family history. The symptoms were progressive: hypesthesia of both hands developed on the seventh day, velopharyngeal insufficiency on the ninth day, and diplopia and ptosis on the tenth day. She was admitted to the hospital two weeks after onset. On examination, severe ataxia of the trunk and limbs were found, and she was unable to 
walk with her eyes closed. Muscle strength was normal. Deep tendon reflexes were absent in the arms and diminished in the legs. Lids were not ptotic but pupils were mydriatic and non-reactive. Convergence was good. Supraduction was slightly impaired. Abduction was restricted bilaterally, which caused diplopia in lateral gaze. Dissociated nystagmus was present in the incompletely abducted eye in both right and left gazes. Rebound nystagmus was noted when the eyes were turned from the left lateral gaze position to the primary position. No positional or positioning nystagmus was observed under Frenzel's glasses. Hyposmia and paresis of the soft palate were noted. Hypesthesia was found in the palms, on the back, and around the mouth. CT showed no abnormality in the brain. Hematological examination and blood chemistry findings were all within normal limits. Antibody titers for influenza and parainfluenza viruses showed no elevation. The peak protein content of the cerebrospinal fluid (CSF) during the course was $79 \mathrm{mg} / \mathrm{dl}$ (normal $<40 \mathrm{mg} / \mathrm{dl}$ ) one month after onset, whereas the cell count was always normal. Hearing acuity and the peripheral nerve conduction velocity were normal. Electronystagmograph (ENG) recordings revealed dissociated nystagmus in lateral gazes and reduced velocity of adduction (Fig. 1) ${ }^{8}$. Caloric responses were normal but the visual suppression of the vestibular nystagmus ${ }^{93}$ was impaired. With oral prednisolone, her symptoms subsided gradually, and she attained full recovery two and a half months after admission.

Case 2: One week after a common cold, a 27-year-old, otherwise healthy woman experienced diplopia and dysequilibrium accompanied by headache. The next day, she became hyperpneic and mentally confused, and she was admitted on the fifth day of onset. She had experienced episodes of hyperventilation syndrome when she was a high school student. Family history was unremarkable. On admission, she was alert but confused. After she became calm by instruction of slow breathing, she no longer complained of headache, but of diplopia. She was unable to walk. On examination, severe ataxia of the trunk and limbs and scanning speech were noted, which indicated cerebellar dysfunction. Muscle strength was normal. Deep tendon reflexes were absent in the limbs. Ptosis was not observed but pupils were mydriatic and the light reflex was sluggish. Convergence was poor. Abduction was restricted bilaterally, causing diplopia. Dissociated nystagmus was present in the abducted eye in both lateral gazes. Pursuit eye movements were not smooth and rebound nystagmus was noted when the eyes were turned from the right lateral to the primary position. No positional or positioning nystagmus was observed under Frenzel's glasses. Hypesthesia was noted in the periphery of limbs and around the lip. CT of the brain was normal. Blood and urine tests were normal and virologic studies were non-contributory. The protein content of the CSF was elevated $(74 \mathrm{mg} / \mathrm{dl})$, but the cell count was within normal limits. Hearing was normal. ENG recordings proved the restricted abduction in right lateral gaze, reduced adduction velocity in rightward and leftward saccades, and overshooting of the abducting left eye (Fig. 2). Caloric responses were normal. Oral prednisolone relieved her symptoms gradually, and she became free of symptoms one and a half months after onset.

Case 3: A 25-year-old woman woke up and found that she had difficulty in upward gaze. The next day, she noticed diplopia in left lateral gaze and photophobia. The diplopia worsened gradually and was noticed in right lateral gaze as well. Finally, she experienced ataxia on the third day, and visited our outpatient clinic one week after the onset. There had been no preceding symptoms of common cold. Past history was not remarkable except for atopic dermatitis. Family history was unremarkable. On examination, her eyes showed bilateral restriction of abduction (Fig. 3a). The red green test of Hess confirmed that the bilateral restriction of abduction caused her diplopia. Vertical eye movement range had become normal by that time. No positional or positioning nystagmus was observed under Frenzel's glasses. The eye movements were recorded with ENG. Dissociated nystagmus of the abducting right eye was observed in the right lateral gaze (Fig. 4a). 

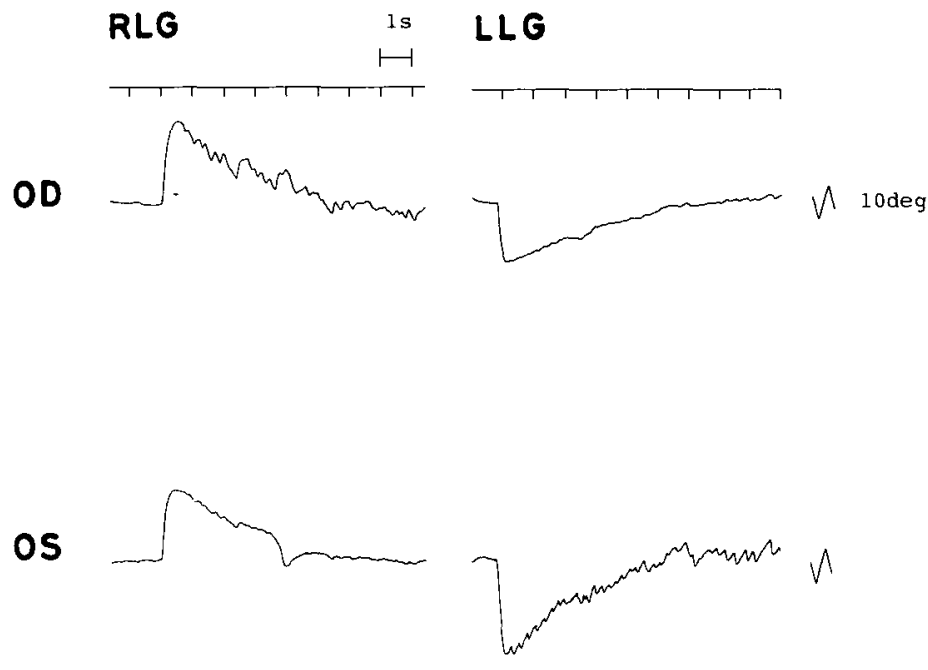

a

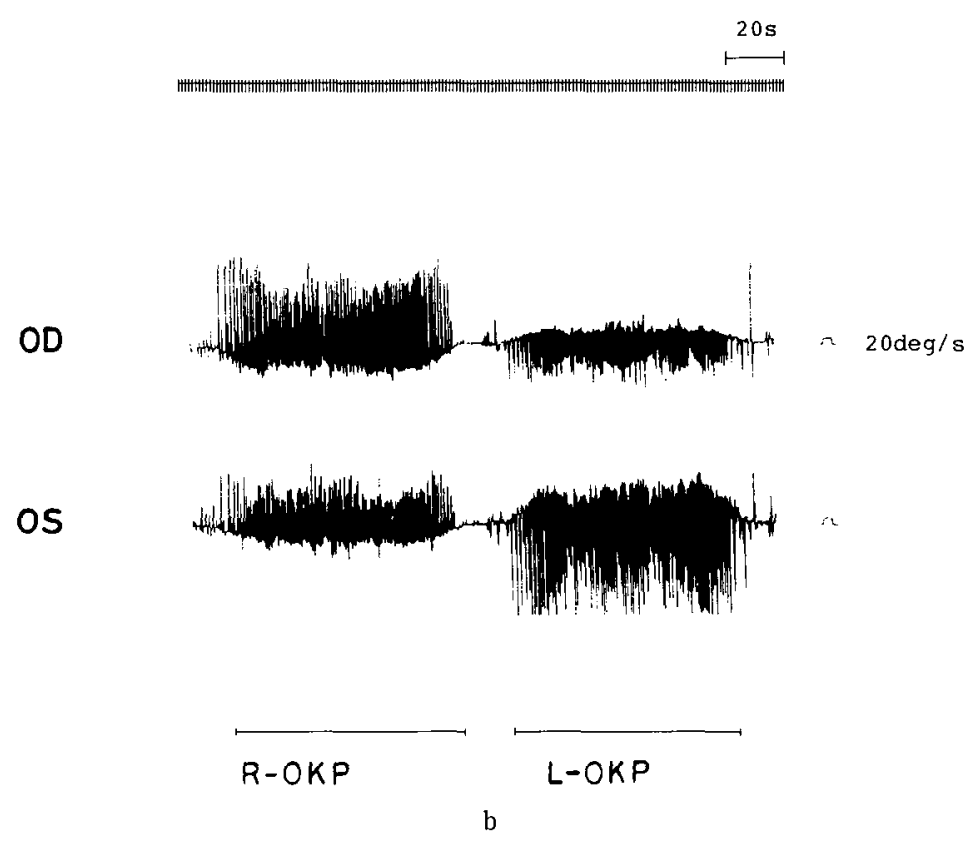

Fig. 1 Electronystagmographic (ENG) recordings of Case 1

OD (oculus dexter) and OS (oculus sinister) represent traces for right and left eyes, respectively. Upward directions signify movements to the right. The uppermost traces indicate the time scale. Eye position and velocity were recorded using $\mathrm{AC}$ modes with time constants of $3 \mathrm{~s}$ and $0.03 \mathrm{~s}$, respectively. a) Eye position traces from the primary position to right lateral gaze (RLG) and left lateral gaze (LLG). Dissociated nystagmus of the abducting eyes were recorded in both lateral gazes. b) Optokinetic pattern tests (OKPs) ${ }^{8}$. Vertical lines in a drum were rotated with an acceleration of $4 \mathrm{deg} / \mathrm{s} / \mathrm{s}$ for $40 \mathrm{~s}$, from 0 to $160 \mathrm{deg} / \mathrm{s}$ in velocity, then decelerated at $-4 \mathrm{deg} / \mathrm{s} / \mathrm{s}$ for $40 \mathrm{~s}$ to a halt. The lines were rotated leftward first, then rightward. Velocity traces are shown. Note that the letters for OKN direction (R, L-OKN) in the figure indicate, by our habit, the direction of evoked optokinetic nystagmus, not the direction of drum rotation. Saccade velocity of both eyes in adduction was reduced. 


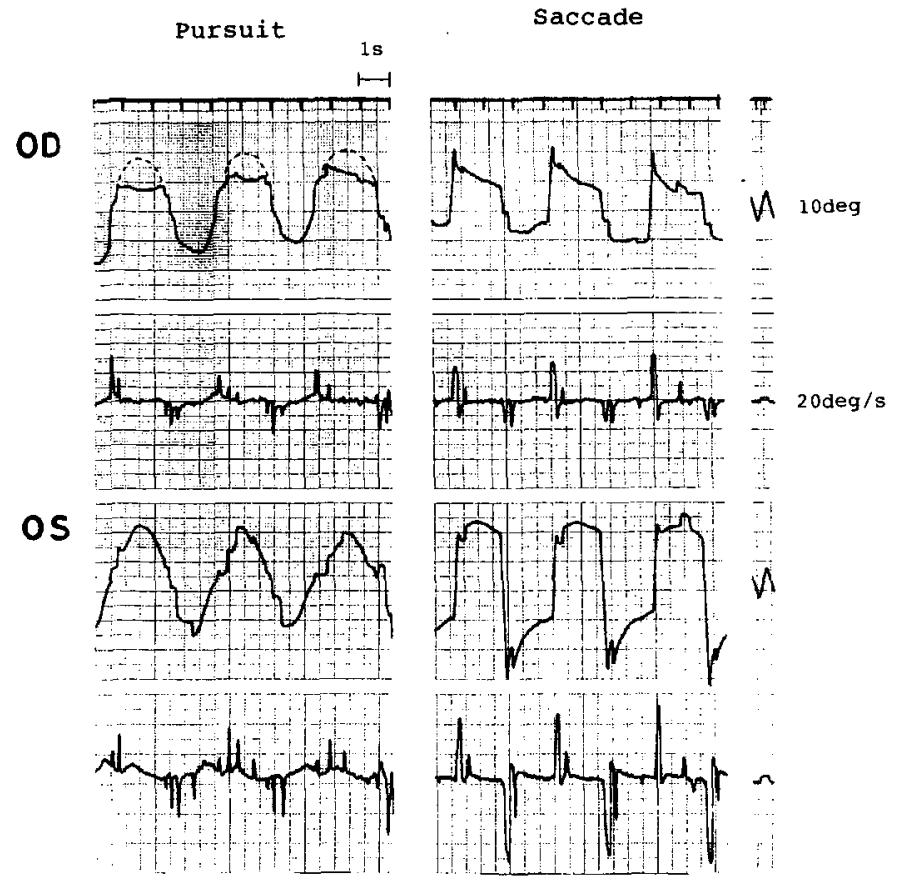

a

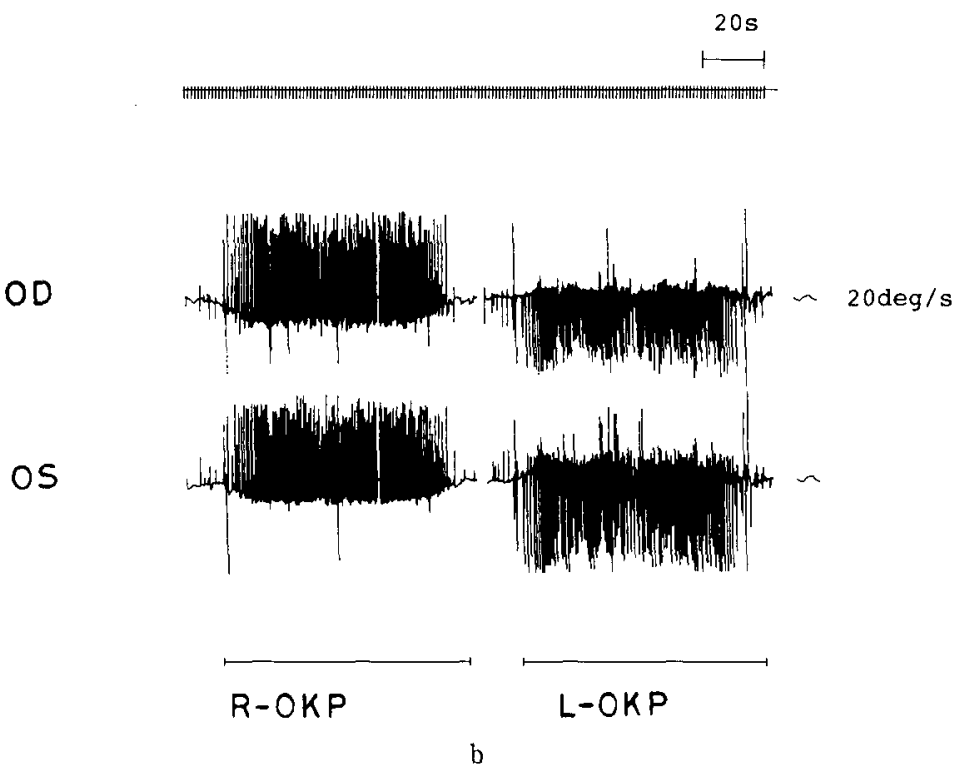

Fig. 2 ENG recordings of Case 2

The recording conditions were the same as in Fig. 1. a) Horizontal pursuits and saccades between two targets. Both position and velocity of the eyes are shown. Restricted abducting range of the right eye is evident in pursuit eye movements. Dotted lines in the recordings represent expected traces in normal subjects. In saccades, reduced adduction velocity of the right eye and overshoots of the left eye in abduction are shown. b) In OKPs, adducting saccade velocity of the right eye is reduced. 


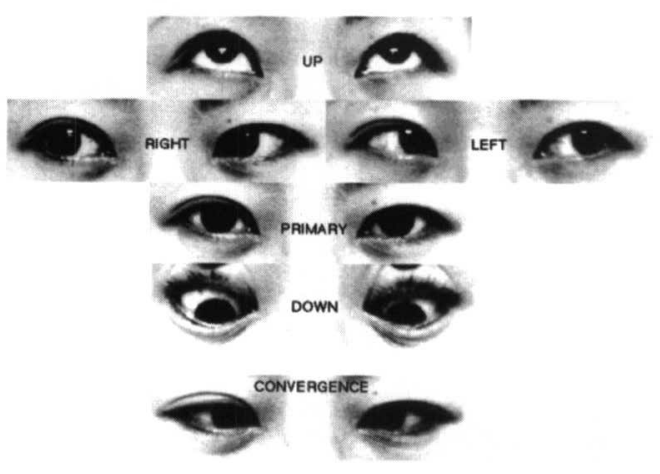

a

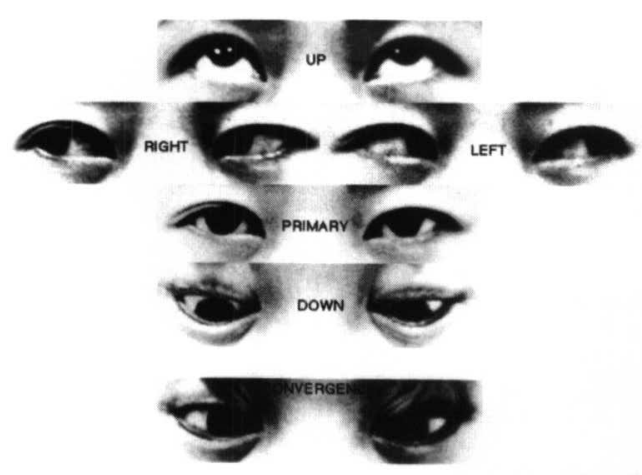

b

Fig. 3 Extent of eye movements in Case 3

a) Eye movements one week after onset. Abduction was restricted bilaterally. b) Eye movements after spontaneous recovery. Abduction ranges have returned to normal.

Saccade velocity was reduced in adduction of the left eye (Fig. 4b). Caloric responses were normal. Her pupils were anisocoric (left>right), mydriatic, and non-reactive. Her hearing was normal. Cranial nerve functions were normal except for the concerning eye movements described. Muscular strength was normal. She showed mild but apparent truncal ataxia. Deep tendon reflexes were absent. Lumber puncture was rejected by the patient. Routine blood tests were negative including anti-viral antibody titers. Her neurological disorders were clinically diagnosed as Fisher's syndrome. Her symptoms gradually subsided without treatment, adducting saccadic velocity of the left eye was improved (Fig. 4b), and abduction ranges became normal (Fig. 3b). No neurological disorders were found two months after onset.

\section{Discussion}

Characteristics of the abnormal eye movements shown above can be summarized as 1) bilateral restriction of the abduction range, 2) bilateral slowing of adduction saccades, and 3) dissociated nystagmus and/or overshooting of the abducting eyes. Although the latter two characteristics are the same as those in INO, the first one is converse to the restricted adduction in INO. Convergence was variable. The abnormalities were observed during the recovery phase of Fisher's syndrome, when disorders of the horizontal eye movements persisted after the vertical movements had become normal. However, this combination of disorders cannot be considered a mere chance association, since it was observed not in a single patient but in three and it persisted for several weeks. The most important point in this combination is the discrepancy of velocity and range in the impairment of duction eye movements. Normally, when an eye movement is impaired, saccade velocity is reduced first, and restriction of the range follows. With our patients, velocity and range seem to be impaired separately; the saccade velocity was impaired only in adduction, and the range of movement was impaired only in abduction. Reports on such eye movement disorders have not been seen in the literature. Hypothetical explanations of the mechanisms underlying these disorders are as follows.

Possible mechanism for the dissociated nystagmus and overshooting

Increased convergence tone, in compensation for impaired adduction, was hypothesized as one of the mechanisms causing the dissociated nystagmus in $\mathrm{INO}^{10)}$. However, this is unlikely to be the cause with our patients, because convergence was impaired in case 2 and because increase in the convergence tone itself may not be expected in the presence of restriction of abduction, rather than adduction.

In cases with INO, dissociated nystagmus and 


\section{RLG}
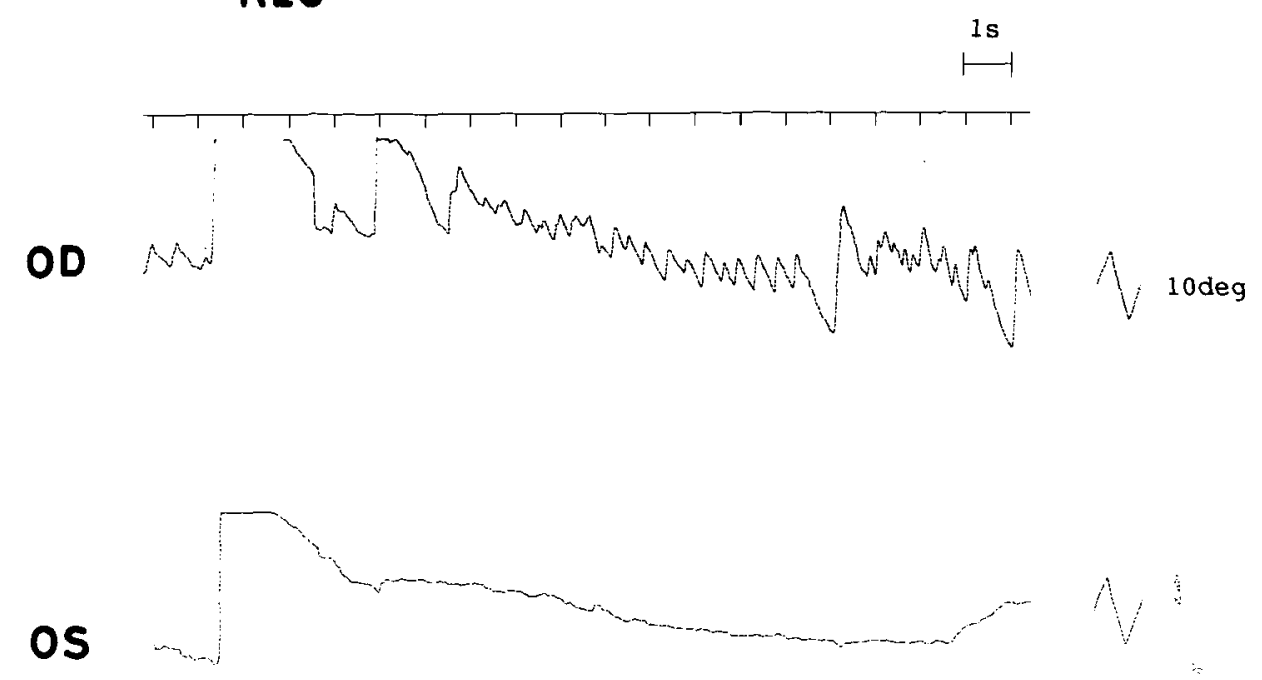

a
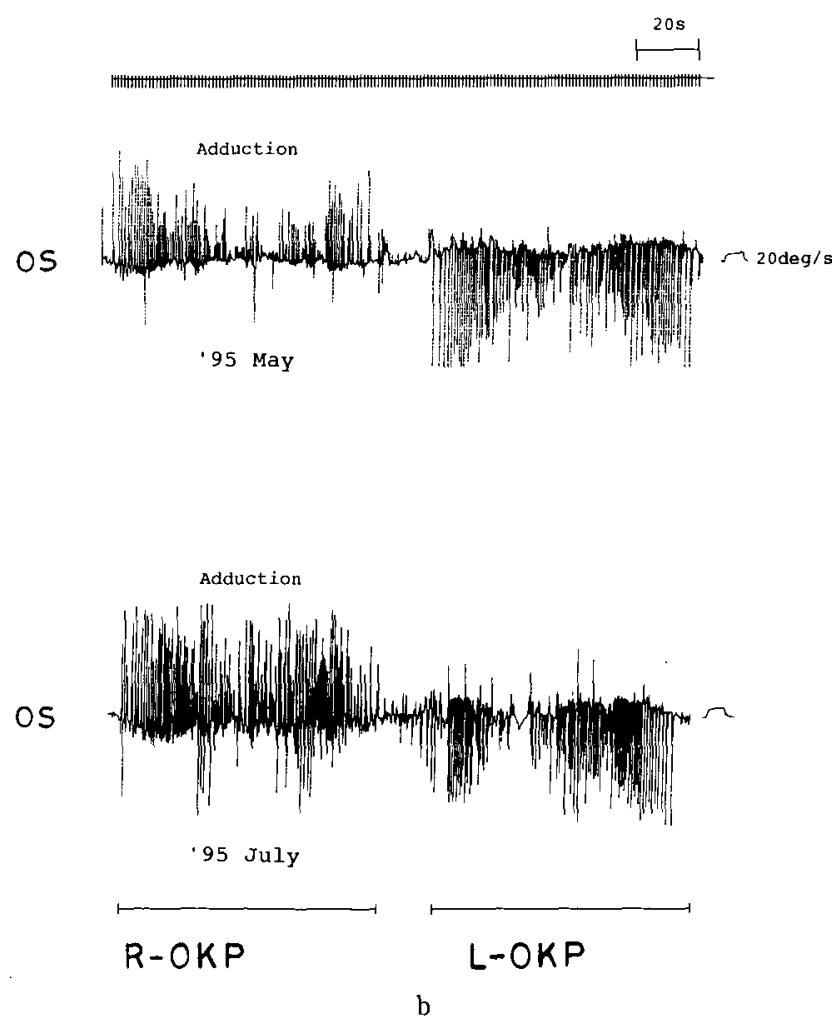

Fig. 4 ENG recordings of Case 3

The recording conditions were the same as in Fig. 1. a) Eye position traces in the right lateral gaze. Dissociated nystagmus of the abducting right eye is shown. b) OKPs revealed improvement of the saccade velocity of the left eye in adduction, in the course of spontaneous recovery. 
overshooting of the abducting eye are explained as results of increased neuronal signals to the abducens muscle, caused by a compensatory mechanism for impairment of adduction. In the case of adduction palsy, increase of signals occurs not only in the medial rectus of the ipsilateral adducting eye but also in the abducens of the contralateral abducting eye, due to Hering's law of equal innervation ${ }^{11}$. In our patients, the conditions were the other way round. As with INO, neuronal signals were increased in both eyes due to Hering's law, as a result of compensatory mechanisms for restricted abduction. The reason why the dissociated nystagmus and overshooting were not observed in the adducting eye but in the abducting eye might be that the preservation of phasic (fast) movements is essential for invoking these abnormal movements. For, in our patients, phasic eye movement was impaired in adduction and tonic (slow) eye movement or eyeholding capability was impaired in abduction.

Possible mechanisms for the interesting combination: restriction of abduction and slowing of adduction

Disinhibition of the medial rectus was hypothesized to explain eye movement disorders in INO by using the pulse-step model ${ }^{12) 13}$, in which lack of inhibition on the medial rectus contralateral to the lesioned MLF caused disturbed, incorrect relationship between the pulse and the step, resulting in abnormal saccades. Computer-simulated abducting saccade model required failure of inhibition in the medial rectus to reproduce the characteristic saccadic trajectories in INO with durations shorter than expected and peak velocities higher than expected for a given saccadic amplitude ${ }^{12}$. This hypothesis can explain the restricted abduction seen in our patients, at least to some extent. From this hypothesis, not only excitation but inhibition is impaired in the medial rectus, disturbing its full relaxation. However, such involvement of supranuclear inhibitory neurons assumes a central disorder in Fisher's syndrome and another problem is that inhibitory neurons running in the $\mathrm{MLF}^{13)}$ are still hypothetical.
An uncommon configuration of pulse-step ${ }^{14)}$ mismatch in abducens neurons will best explain the eye movement disorders in our patients, although it is unusual as peripheral nerve dysfunction. Normally, pulse signals (high frequency signals which cause phasic, rapid eye movement) are impaired first, whereas step signals (low frequency signals which keep the eye in a position) are relatively preserved in the demyelination of peripheral nerves. As a result, saccade velocity reduces first, and then the restricted range of eye movement becomes manifest. However, if the step signals were significantly deteriorated in contrast to the preserved pulse signals in the abducens muscle and the pulse signals were more deteriorated in the medial rectus in a usual manner, the uncommon combination of restricted abduction and slow adduction may occur. Moreover, using this explanation, everything can be accounted for as peripheral disorders, with certain central compensations for them. As an anatomical counterpart for this hypothesis, separate locations of motoneurons innervating slow (non-twitch) and fast (twitch) extraocular muscle fibers have recently been identified in monkeys ${ }^{15)-17}$, suggesting independent control of fast (phasic) and slow (tonic) eye movements in the peripheral level.

Is this disorder peripheral or central ?

Purely peripheral disorders, such as myasthenia gravis $^{2)}$ or medial rectus muscle damage ${ }^{18)}$, can cause INO-like eye movements (pseudo-INO). Although ocular palsies in Fisher's syndrome were originally classified as purely peripheral ${ }^{19}$, central dysfunctions in Fisher's syndrome have also been reported ${ }^{6 / 7)}$. If the hypothesized inhibitory pathway in the MLF exists, the conditions in our patients may be purely supranuclear (impairment of both excitatory and inhibitory pathways in the MLF) or a mixture of peripheral (impairment of motoneurons innervating the medial rectus) and supranuclear (impairment of the inhibitory pathway in the MLF) disorders. However, if independent impairment of phasic and tonic movements of the extraocular muscles were possible in peripheral neuropathy, as described above, pure peripheral disorders, with 
central compensations, can explain the disorders. Since the latter explanation is more simple and is less hypothetical in that it has anatomical counterparts, purely peripheral involvement would be more suitable for explaining the conditions of our patients.

After the first report in $1992^{20)}$, anti-GQ1b IgG antiganglioside antibody has gained the position of a highly specific marker for Fisher's syndrome ${ }^{21}$. Immunohistochemical studies showed existence of GQ1b in the extramedullary portions of human $3^{\text {rd }}$, $4^{\text {th }}$, and $6^{\text {th }}$ cranial nerves ${ }^{22)}$ as well as the molecular layer of the human cerebellum ${ }^{23}$. Unfortunately, our patients were examined before screening for the anti-GQ1b IgG became prevalent, and therefore the diagnosis was made from the clinical point of view. However, since existence or absence of a substance shown by immunohistopathological methods may not necessarily be related to the function and since there has not been a report in which the clinical and pathologic features of Fisher's syndrome was reproduced by passive transfer of this antibody ${ }^{21}$, measurement of serum anti-GQ1b antibody titer cannot give clues to the detailed functional evaluation, e.g. determination of peripheral or central disorders.

\section{Conclusions}

In this paper, three cases were reported that showed peculiar but interesting eye movement disorders in the course of Fisher's syndrome, i.e., INO-like eye movements in the presence of abduction restriction. Possible impairment of inhibition to the medial rectus and independent peripheral control of phasic and tonic eye movements were hypothesized in order to account for this set of disorders. The latter explanation was considered more suitable. Since there still remains much to be revealed concerning the mechanisms controlling monocular eye movements, further accumulation of interesting cases will be of help.

\section{Acknowledgements}

Part of this paper was presented in an abstract form at the 20th meeting of the Bárány Society, Würtzburg, Germany, 1998²4.

\section{References}

1) Cogan DG: Internuclear ophthalmoplegia, typical and atypical. Arch Ophthalmol 84: 583-589, 1970

2) Glaser JS: Myasthenic pseudo-internuclear ophthalmoplegia. Arch Ophthalmol 75: 363366, 1966

3) Ito K, Mizutani J, Murofushi T, et al: Bilateral pseudo-internuclear ophthalmoplegia in myasthenia gravis. ORL J Otorhinolaryngol Relat Spec 59: 122-126, 1997

4) Zasorin $\mathrm{NL}$, Yee RD, Baloh RW: Eye-movement abnormalities in ophthalmoplegia, ataxia, and areflexia (Fisher's syndrome). Arch Ophthalmol 103: 55-58, 1985

5) Swick HM: Pseudointernuclear ophthalmoplegia in acute idiopathic polyneuritis (Fischer's syndrome). Am J Ophthalmol 77: 725-728, 1974

6) Meienberg O, Ryffel E: Supranuclear eye movement disorders in Fisher's syndrome of ophthalmoplegia, ataxia, and areflexia. Report of a case and literature review. Arch Neurol 40: 402-405, 1983

7) Toshniwal P: Demyelinating optic neuropathy with Miller-Fisher syndrome. The case for overlap syndromes with central and peripheral demyelination. J Neurol 234: 353-358, 1987

8) Suzuki J, Komatsuzaki A: Clinical application of optokinetic nystagmus: Optokinetic pattern test. Acta Otolaryngol 54: 49-55, 1962

9) Takemori S: Visual suppression test. Ann Otol Rhinol Laryngol 86(1 Pt 1): 80-85, 1977

10) Stroud MH, Newman NM, Keltner JL, et al: Abducting nystagmus in the medial longitudinal fasciculus (MLF) syndrome (internuclear ophthalmoplegia [INO]). Adv Otorhinolaryngol 19: 367-376, 1973

11) Hering E: The theory of binocular vision. In eds Bridgeman B, Stark L. Plenum Press, New York, 1977

12) Feldon SE, Hoyt WF, Stark L: Disordered inhibition in internuclear ophthalmoplegia: analysis of eye movement recordings with computer 
simulations. Brain 103: 113-137, 1980

13) Pola J, Robinson DA: An explanatioin of eye movements seen in internuclear ophthalmoplegia. Arch Neurol 33: 447-452, 1976

14) Robinson DA: Oculomotor control signals. In eds Lennerstrand G, Bach-y-Rita P. Basic mechanisms of ocular motility and their clinical implications. pp 337-374, Pergamon Press, Oxford, 1975

15) Buttner-Ennever JA, Horn AKE, Scherberger $\mathrm{H}$, et al: The location of motoneurons innervating slow and fast extraocular eye muscle fibres in monkey. In eds Pompeiano O, Wilson V. Satellite Symposium to the XXth Meeting of the Bárány-Society. pp12, Freiburg, Germany, 1998

16) Buttner-Ennever JA, Horn AK: The neuroanatomical basis of oculomotor disorders: the dual motor control of extraocular muscles and its possible role in proprioception. Curr Opin Neurol 15: 35-43, 2002

17) Buttner-Ennever JA, Horn AK, Scherberger $H$, et al: Motoneurons of twitch and nontwitch extraocular muscle fibers in the abducens, trochlear, and oculomotor nuclei of monkeys. J Comp Neurol 438: 318-335, 2001

18) von Noorden GK, Tredici TD, Ruttum M: Pseudo-internuclear ophthalmoplegia after surgical paresis of the medial rectus muscle. Am J Ophthalmol 98: 602-608, 1984

19) Fisher M: An unusual variant of acute idiopathic polyneuritis (syndrome of ophthalmople- gia, ataxia and areflexia). $\mathrm{N}$ Engl J Med 255: 57 $-65,1956$

20) Chiba A, Kusunoki S, Shimizu T, et al: Serum IgG antibody to ganglioside GQ1b is a possible marker of Miller Fisher syndrome. Ann Neurol 31: 677-679, 1992

21) Paparounas K: Anti-GQ1b ganglioside antibody in peripheral nervous system disorders: pathophysiologic role and clinical relevance. Arch Neurol 61: 1013-1016, 2004

22) Chiba A, Kusunoki S, Obata $H$, et al: Serum anti-GQ1b IgG antibody is associated with ophthalmoplegia in Miller Fisher syndrome and Guillain-Barre syndrome: clinical and immunohistochemical studies. Neurology 43: 19111917, 1993

23) Kornberg AJ, Pestronk A, Blume GM, et al: Selective staining of the cerebellar molecular layer by serum IgG in Miller-Fisher and related syndromes. Neurology 47: 1317-1320, 1996

24) Ito K, Mizuno M, Murofushi T, et al: Dissociated nystagmus, restricted abduction, and reduced adduction velocity - a strage combination observed in Fisher's syndrome. Int Tinnitus J 4 Suppl 1: 28, 1998

原稿到着: 平成 17 年 2 月 28 日

別刷請求先：伊藤 健

干113-8655 東京都文京区本郷7-3-1

東京大学医学部耳鼻咽喉科

E-mail: itoken-tky@umin.ac.jp 